O. FRAZÃO ${ }^{1, \square}$

J.M. MARQUES ${ }^{1}$

J.L. SANTOS ${ }^{1,3}$

M.B. MARQUES ${ }^{1,3}$

J.M. BAPTISTA ${ }^{1,2}$

\title{
Brillouin fibre laser discrete sensor for simultaneous strain and temperature measurement
}

${ }^{1}$ INESC Porto, Rua do Campo Alegre 687, 4169-007 Porto, Portugal

2 Dep. de Matemática e Engenharias, Universidade da Madeira, Campus da Penteada, 9000-390 Funchal, Portugal

${ }^{3}$ Dep. de Física, FCUP, Rua do Campo Alegre 687, 4169-007 Porto, Portugal

\section{Received: 24 May 2006/Revised version: 31 October 2006} Published online: 19 December 2006 • () S Springer-Verlag 2006

ABSTRACT In this work, a Brillouin fibre laser sensor for strain and temperature discrimination is presented. The fibre laser sensor consists of a Fabry-Pérot cavity with $20 \mathrm{~m}$ of optical fibre between two Bragg gratings. For the strain measurement, the $20 \mathrm{~m}$ were split in half and in $10 \mathrm{~m}$ a pre-tension was applied originating two Brillouin peaks. For the temperature measurement all of the sensing head was heated. The resolutions achieved were $\pm 1 \mu \varepsilon$ and $\pm 1{ }^{\circ} \mathrm{C}$ for strain and temperature measurements, respectively.

PACS 42.81.-I; 42.55.Wd; 42.65.Es

\section{1}

\section{Introduction}

The scattering effects in optical fibres consist of elastic or inelastic scattering, where the wavelengths of the incident and scattered light are the same, or differ from each other, respectively. Brillouin scattering is a type of inelastic scattering, where the wavelength shift depends on sound velocity in the fibre. Due to this characteristic, the Brillouin wavelength is shifted when strain and/or temperature changes.

Several authors have proposed different techniques for simultaneous measurement of strain and temperature. The most common technique is to measure the change of Brillouin peak intensity and frequency. For instance, Parker et al. used a sensing length of $1200 \mathrm{~m}$ [1], Smith et al. proposed and demonstrated a technique based on the Brillouin loss mechanism using a sensing length of $50 \mathrm{~m}$ of fibre [2]. Other works are based on large-effective-area fibre, which has a multiple composition fibre core. This type of fibre can generate multiple Brillouin peaks providing also a possible discrimination method for strain and temperature measurement [3].

On the other hand, several configurations for Brillouin lasers have been proposed [4,5]. Traditionally two configurations of fibre laser sensors can be used. One of them is based on a ring geometry [4], others consist in a Fabry-Pérot cavity

Fax: +35122 6082799, E-mail: ofrazao@inescporto.pt using two mirrors (for example, two FBGs) [5]. The advantage of the second solution when compared with the first one is having a smaller cavity length. In that work, Shen et al., demonstrated the generation of two wavelengths in a $10 \mathrm{~m}$ Brillouin fibre laser based on Bragg grating technology for an all optical generation of microwave and millimetre wave [5].

In this work, we propose a Brillouin fibre laser sensor for simultaneous measurement of strain and temperature with a sensing length of $20 \mathrm{~m}$. For the temperature measurement, all the fibre of the laser cavity is placed in a oven. For the strain measurement half of the fibre length of the laser cavity is pre-tensioned and subjected to strain variation, while the other half is used as reference. In this case, the laser oscillates simultaneously in two sets of frequencies, being one set related to one half of the fibre, and the other set related to the other half of the fibre.

2

\section{Principle}

A theoretical analysis of cascaded stimulated Brillouin scattering (CSBS), in a fibre Fabry-Pérot resonator with two fibre Bragg gratings (FBG), is presented.

The first Stokes wave will be generated by the pump wave that passes through $\mathrm{FBG}_{1}$, with a reflectivity $R_{1}$. Then the pump will be reflected, in $\mathrm{FBG}_{2}$ with a reflectivity $R_{2}$ (see Fig. 1). This continuous process of the reflections in the resonator generates forward and backward propagation of the pump, making possible the production of higher order Stokes. Neglecting the phase of each Stokes wave in the coupledamplitude equations, we derive the following:

$$
\begin{aligned}
& \frac{\partial P_{\mathrm{p}}^{ \pm}(z)}{\partial z}=\mp \alpha P_{\mathrm{p}}^{ \pm}(z) \mp \frac{g_{\mathrm{B}}}{A_{\mathrm{eff}}} P_{\mathrm{s} 1}^{\mp}(z) P_{\mathrm{p}}^{ \pm}(z) \\
& \frac{\partial P_{\mathrm{s} 1}^{ \pm}(z)}{\partial z}=\mp \alpha P_{\mathrm{s} 1}^{ \pm}(z) \pm \frac{g_{\mathrm{B}}}{A_{\mathrm{eff}}}\left(P_{\mathrm{p}}^{\mp}(z)-P_{\mathrm{s} 2}^{\mp}(z)\right) P_{\mathrm{s} 1}^{ \pm}(z) \\
& \vdots \\
& \frac{\partial P_{\mathrm{s} N}^{ \pm}(z)}{\partial z}=\mp \alpha P_{\mathrm{s} N}^{ \pm}(z) \pm \frac{g_{\mathrm{B}}}{A_{\mathrm{eff}}}\left(P_{\mathrm{s}(N-1)}^{\mp}(z)\right) P_{\mathrm{s} N}^{ \pm}(z)
\end{aligned}
$$

where $P_{\mathrm{P}}(z)$ and $P_{\mathrm{s} i}(z), i=1,2, \ldots, N$, represent the powers of pump and $i$-th Stokes waves, respectively. The direction of 


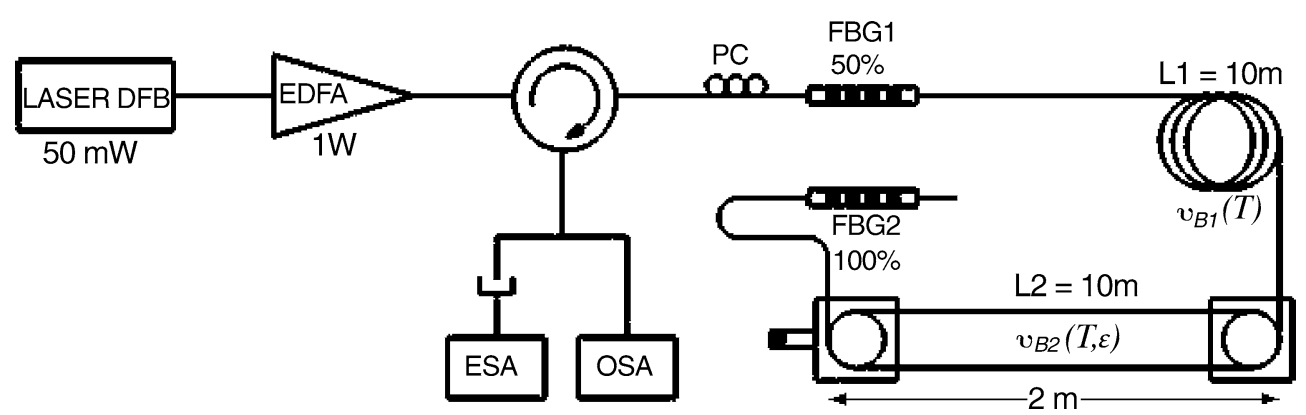

FIGURE 1 Experimental setup of the Brillouin laser sensor

propagation is indicated by ' \pm ', being the propagation from the $\mathrm{FBG}_{1}$ to $\mathrm{FBG}_{2}$ designated by the sign ' + '.$\alpha$ indicates the fibre attenuation, $g_{\mathrm{B}}$ the Brillouin gain and $A_{\text {eff }}$ the fibre effective core area. At the gratings there are different boundary conditions, at $\mathrm{FBG}_{1}(z=0)$ and at $\mathrm{FBG}_{2}(z=L)$, resulting in:

$P_{\mathrm{p}}^{+}(0)=\left(1-R_{1}\right) P_{\mathrm{in}}+R_{1} P_{\mathrm{p}}^{-}(0)$

$P_{\mathrm{s} i}^{+}(0)=R_{1} P_{\mathrm{s} i}^{-}(0) \quad(i=1,2, \ldots, N)$

$P_{\mathrm{p}}^{-}(L)=R_{2} P_{\mathrm{p}}^{+}(L)$

$P_{\mathrm{s} i}^{-}(L)=R_{2} P_{\mathrm{s} i}^{+}(L) \quad(i=1,2, \ldots, N)$,

where $P_{\text {in }}$ is the pump power, $\left(1-R_{1}\right) P_{\text {in }}$ the optical power that passes through $\mathrm{FBG}_{1}$ and $R_{k} P_{n}$, with $k=1,2$; and $n=s i$, $p$; represents the reflection on a FBG by Stokes or pump wave respectively. The numeric results are demonstrated in [6].

\section{$3 \quad$ Experimental setup}

The experimental setup is depicted in Fig. 1. A DFB diode laser, controlled in current and temperature with maximum power of $50 \mathrm{~mW}$, central wavelength of $1554.15 \mathrm{~nm}$ and linewidth of $1 \mathrm{MHz}$ is used. It is followed by an erbium-doped fibre amplifier (EDFA), IPG laser model EAD-1K-C3-W, of $1 \mathrm{~W}$ of maximum amplification. After the optical circulator a polarization controller $(\mathrm{PC})$ is used to control the polarization of the incident wave. The Brillouin laser sensor consists of a Fabry-Pérot cavity, with 20 m of SMF 28 ,

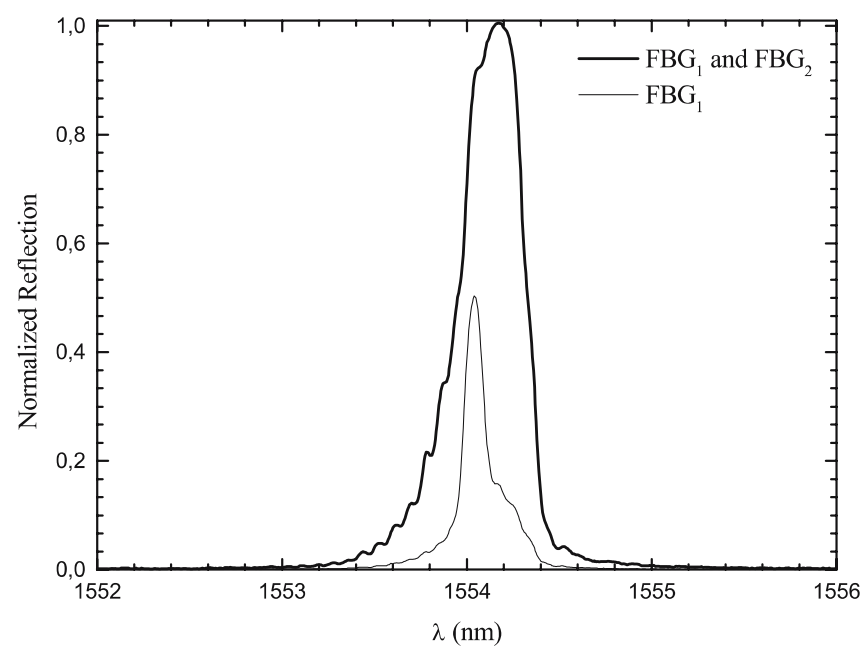

FIGURE 2 Reflection spectra of $\mathrm{FBG}_{1}$ and the combined spectra of the two FBGs between two FBGs. The launched cw optical power into the laser cavity was approximately $750 \mathrm{~mW}$. The following are the gratings' characteristics: for $\mathrm{FBG}_{1} \lambda_{\mathrm{B} 1}=1554.042 \mathrm{~nm}$, bandwidth $=0.125 \mathrm{~nm}$ and reflectivity $\sim 50 \%$; for $\mathrm{FBG}_{2}$ $\lambda_{\mathrm{B} 2}=1554.137 \mathrm{~nm}$, bandwidth $=0.375 \mathrm{~nm}$ and reflectivity $\sim 100 \%$ as can be seen in Fig. 2. The bandwidth of $\mathrm{FBG}_{1}$ must be narrower than $v_{\mathrm{b}}$ (acoustic phonon frequency) to generate first order Stokes only and, consequentially, zero reflectivity for higher orders. This grating is used as pump dichroic mirror and laser output mirror. $\mathrm{FBG}_{2}$ has nearly full reflectivity in order to reflect both Stokes and pump waves. The Stokes wave is amplified along the cavity path, which consists in $20 \mathrm{~m}$.

The optical signal is measured in reflection and is read by an optical spectrum analyser (OSA) with a maximum resolution of $0.05 \mathrm{~nm}$ and the optical detector used with the electric spectrum analyser (ESA) was an HP 11981 A lightwave converter $1200-1600 \mathrm{~nm}$ with a bandwidth of $12 \mathrm{GHz}$. To maximize the beat discrimination the gratings were optimized in order to have the same output peak power of the Brillouin and pump signal.

\section{$4 \quad$ Experimental results}

Figure 3 shows the Brillouin fibre laser output signal, the pump and Brillouin signals have central wavelengths of $1553.98 \mathrm{~nm}$ and $1554.07 \mathrm{~nm}$, respectively. After optimization, the beat of each frequency of the laser was observed in the ESA with a resolution of $100 \mathrm{kHz}$. Figure 4 shows that result, where it can be seen that the longitudinal modes are

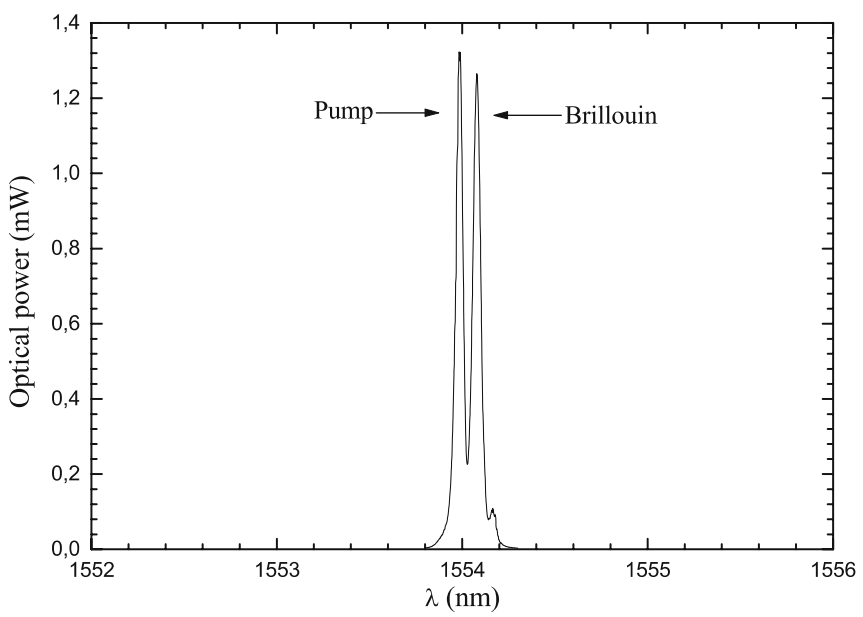

FIGURE 3 Output signal of the Brillouin fibre laser 


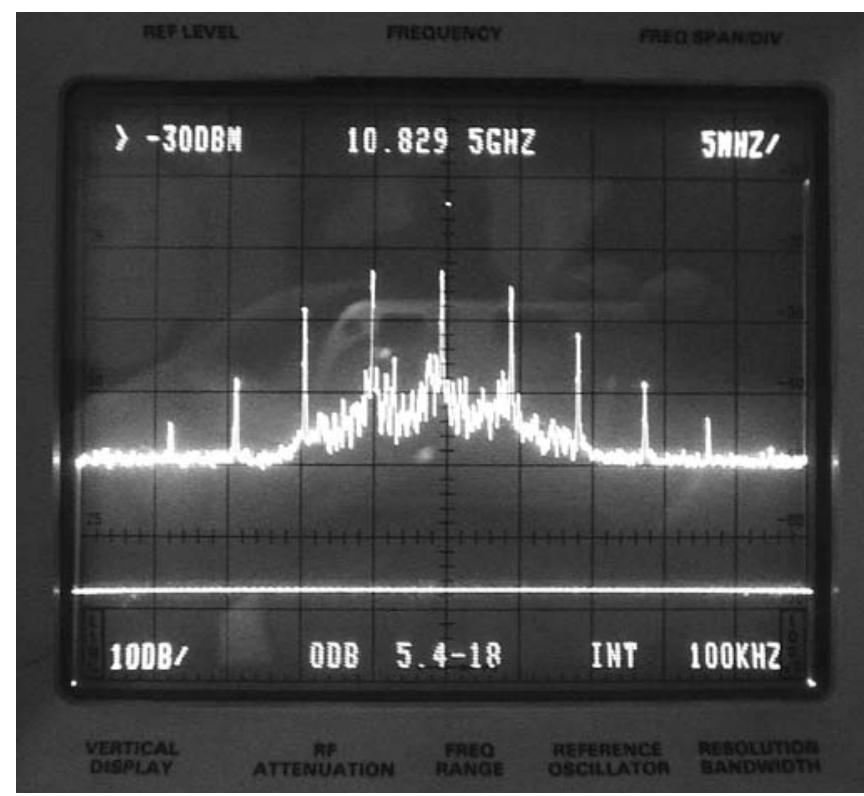

FIGURE 4 Longitudinal modes of the Brillouin fibre laser

separated by $\approx 5.108 \mathrm{MHz}$. The linewidth of the Brillouin spectrum is approximately $35 \mathrm{MHz}$.

As mentioned earlier, the sensing head consists of $20 \mathrm{~m}$ of fibre length divided in two equal parts $(10 \mathrm{~m}$ each). For the temperature measurement all the $20 \mathrm{~m}$ were subjected to temperature variation. For the strain measurement, a pre-tension $(\sim 200 \mu \varepsilon)$ was applied to $10 \mathrm{~m}$ to divide the Brillouin signal in $v_{\mathrm{B} 1}$ and $v_{\mathrm{B} 2} . v_{\mathrm{B} 1}$ corresponds to the $10 \mathrm{~m}$ without strain and $v_{\mathrm{B} 2}$ corresponds initially to the pre-tensioned fibre and varies accordingly with the applied strain. $v_{\mathrm{B} 1}$ and $v_{\mathrm{B} 2}$ have values of $10.828 \mathrm{GHz}$ and $10.844 \mathrm{GHz}$, respectively (Fig. 5).

The representation of the Brillouin frequency variation when strain is applied, for room temperature $\left(20^{\circ} \mathrm{C}\right)$, can be observed in Fig. 6. The slope of $v_{\mathrm{B} 2}$ is $0.072 \pm$

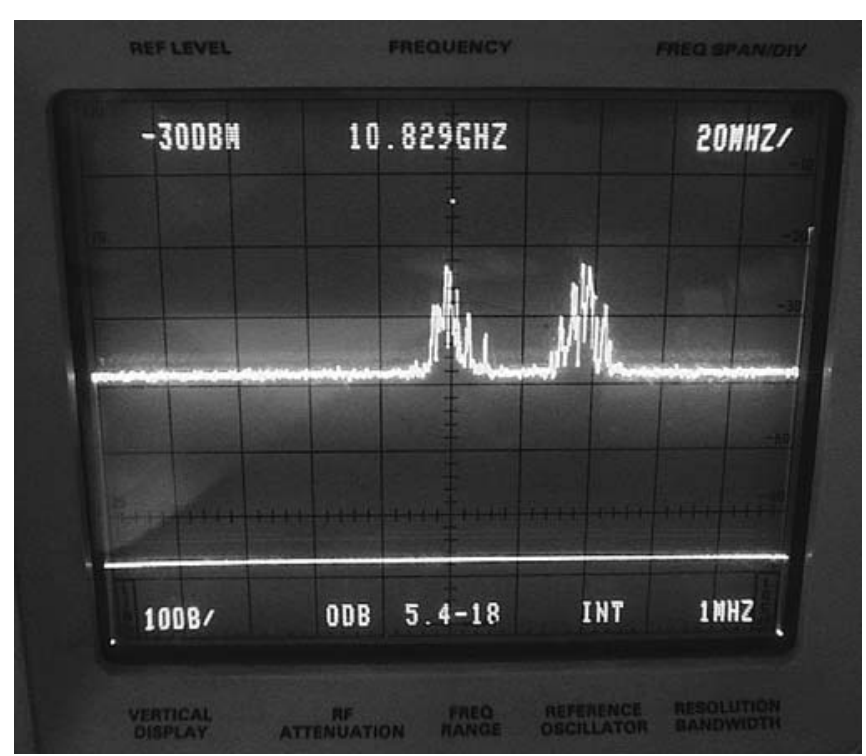

FIGURE $5 \quad v_{\mathrm{B} 1}$ and $v_{\mathrm{B} 2}$ Brillouin signals ( $v_{\mathrm{B} 2}$ results from the pretensioned fibre and varies accordingly with applied strain)

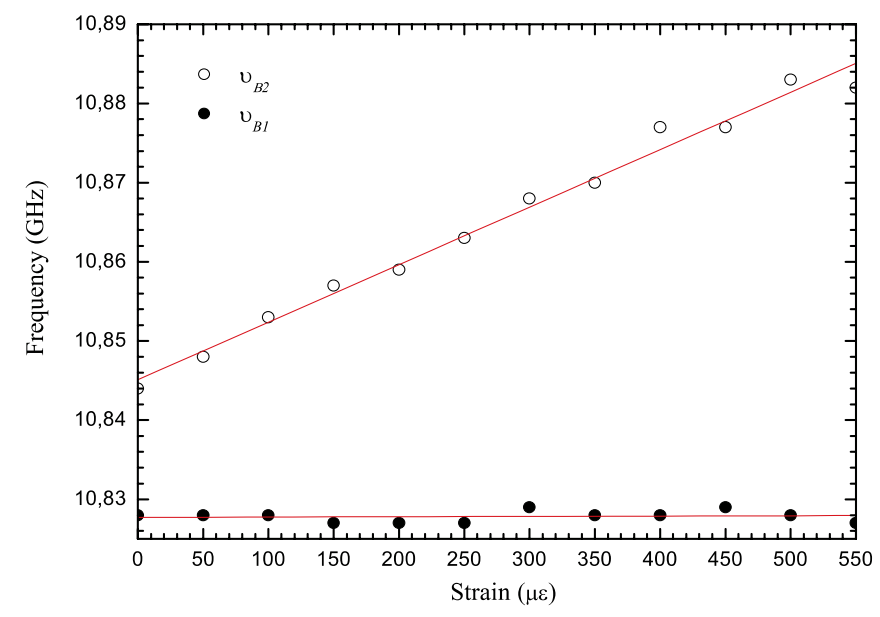

FIGURE 6 Response of the Brillouin fibre laser to strain variation

$0.002 \mathrm{MHz} / \mu \varepsilon$, and is close to the value reported in [7], which is $0.055 \mathrm{MHz} / \mu \varepsilon$.

The second step consisted in the characterization of the sensing head when it was subjected to temperature variation with no strain applied. All of the $20 \mathrm{~m}$ of the Brillouin laser sensor cavity were heated between $20^{\circ} \mathrm{C}$ and $100^{\circ} \mathrm{C}$, and it was possible to observe the variation of the Brillouin peak frequency with temperature change (Fig. 7). In this experiment only one Brillouin peak was observed because the fibre inside the cavity did not have different tensions applied so, in this case $v_{\mathrm{B} 1}=v_{\mathrm{B} 2}$. As shown in Fig. 7, the slope was $1.0 \pm 0.02 \mathrm{MHz} /{ }^{\circ} \mathrm{C}$, also comparable with the value of $1.69 \mathrm{MHz} /{ }^{\circ} \mathrm{C}$, given in [7].

When temperature and strain applied to the sensing head change, one of the frequencies is shifted according to $\Delta v_{\mathrm{B} i}=$ $C_{\mathrm{T} i} \Delta T+C_{\varepsilon i} \Delta \varepsilon$, where $\Delta T$ and $\Delta \varepsilon$ are temperature and strain variation, respectively and $i=1,2$. This permits to write in principle a well-conditioned system of two equations for $\Delta T$ and $\Delta \varepsilon$ given in a matrix form as:

$\left[\begin{array}{l}\Delta T \\ \Delta \varepsilon\end{array}\right]=\frac{1}{D}\left[\begin{array}{ll}C_{\varepsilon 2} & -C_{\varepsilon 1} \\ -C_{\mathrm{T}} & C_{\mathrm{T}}\end{array}\right]\left[\begin{array}{l}\Delta v_{\mathrm{B} 1} \\ \Delta v_{\mathrm{B} 2}\end{array}\right]$

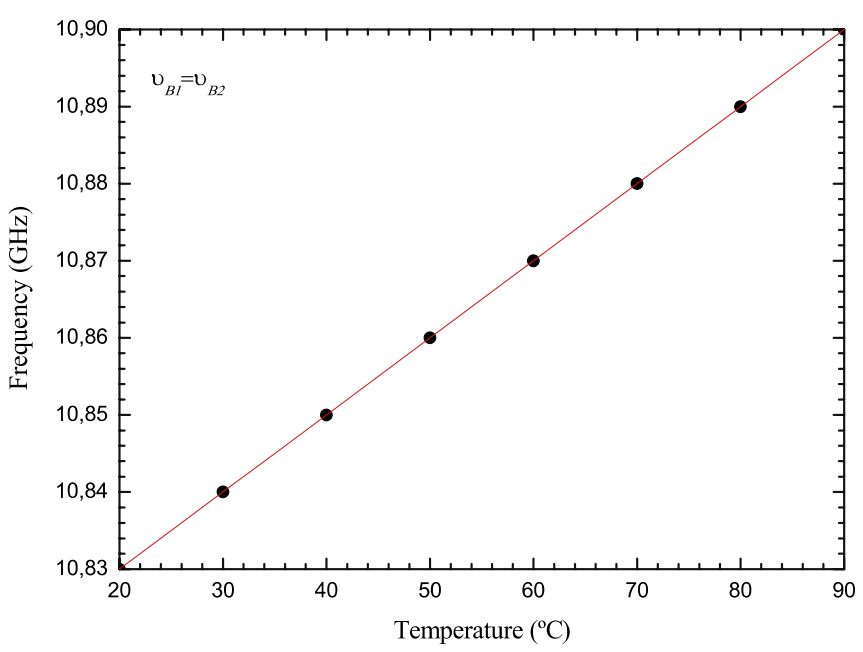

FIGURE 7 Response of the Brillouin fibre laser to temperature variation 


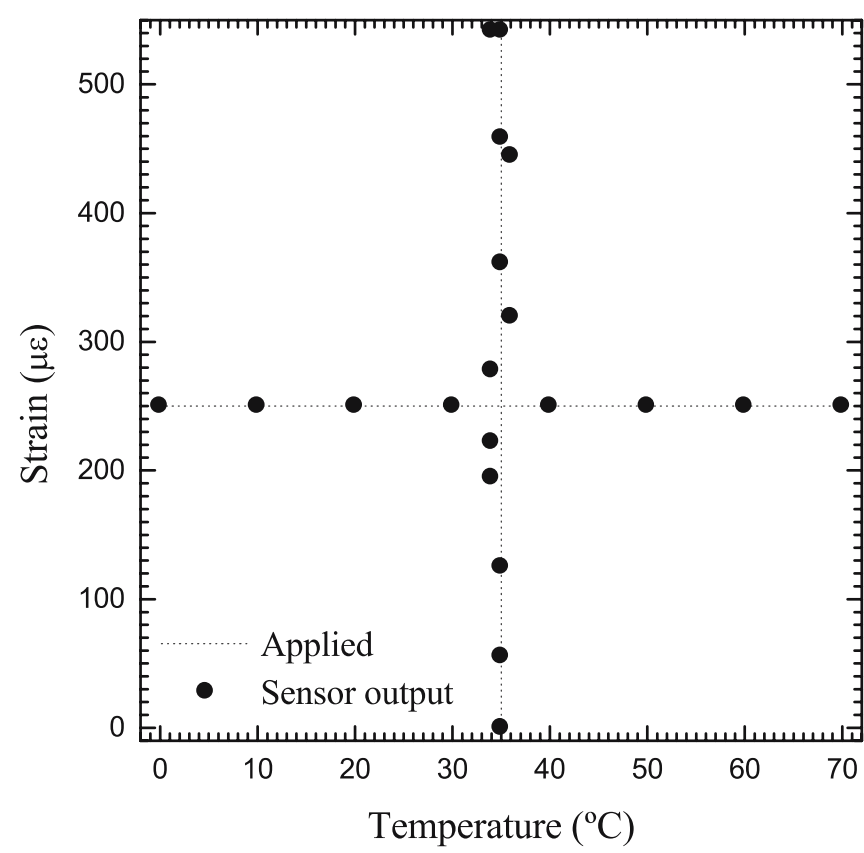

FIGURE 8 Sensor output as determined by (4) for applied strain at constant temperature and applied temperature at constant strain

where the determinant is $D=C_{\mathrm{T}} C_{\varepsilon 2}-C_{\varepsilon 1} C_{\mathrm{T}}$. The strain sensitivities $\left(C_{\varepsilon 1}, C_{\varepsilon 2}\right)$ and thermal sensitivity $\left(C_{\mathrm{T}}\right)$ are the sensor head sensitivity coefficients. The matrix coefficients are obtained from the experimental slopes shown in Figs. 6 and 7, resulting in:

$$
\left[\begin{array}{l}
\Delta T \\
\Delta \varepsilon
\end{array}\right]=\frac{1}{0.072}\left[\begin{array}{ll}
0.072 & 0 \\
-1 & 1
\end{array}\right]\left[\begin{array}{l}
\Delta v_{\mathrm{B} 1} \\
\Delta v_{\mathrm{B} 2}
\end{array}\right]
$$

with $\Delta v_{\mathrm{B}}$ in $\mathrm{MHz}, \Delta T$ in ${ }^{\circ} \mathrm{C}$ and $\Delta \varepsilon$ in $\mu \varepsilon$ (microstrain).

The sensor resolution was determined when the sensing head was subject to strain and temperature changes with ranges of $550 \mu \varepsilon$ and $90^{\circ} \mathrm{C}$, respectively. From (4) it is pos- sible to obtain the result in Fig. 8. The data broadening in that figure indicates rms deviations of $\pm 1 \mu \varepsilon$ and $\pm 1{ }^{\circ} \mathrm{C}$ for strain and temperature measurements, respectively.

\section{5}

\section{Conclusion}

It was proposed and demonstrated a Brillouin fibre laser sensing configuration for strain and temperature discrimination. The relatively short cavity length $(20 \mathrm{~m})$ makes the compact sensing head, which is adequate for point measurements, accessible. The resolutions achieved were $\pm 1 \mu \varepsilon$ and $\pm 1{ }^{\circ} \mathrm{C}$ for strain and temperature measurements, respectively, which are appropriated in many applications. This sensor allows higher strain measurement range when compared to other fibre optic sensors which require modification of the optical fibre, such as the FBGs. Wavelength multiplexing is compatible with this sensing concept by associating each sensing head of a pair of gratings with specific Bragg wavelengths. This characteristic, to be explored further in the future, increases the relevance of the described laser structure in the context of fibre optic sensing. This discrete sensor can be used in many applications, namely, in construction (bridge, tunnels), aeronautic and energy (wind, nuclear and oil and gas).

ACKNOWLEDGEMENTS This work was supported in part by "Fundaçao para a Ciência e Tecnologia" under the programme "Programa Operacional Ciência Tecnologia e Inovação"-POCTI/FEDER with grant REEQ/1272/EEI/2005 Fibre Optic Supported Broadband Communication Networks.

\section{REFERENCES}

1 T.R. Parker, M. Farhadiroushan, V.A. Handerek, A.J. Rogers, IEEE Photon. Technol. Lett. 9, 7 (1997)

2 J. Smith, A. Brown, M. DeMerchant, X. Bao, Appl. Opt. 38, 25 (1999)

3 M. Alahbabi, Y.T. Choo, T.P. Newson, Opt. Lett. 29, 1 (2004)

4 P.-A. Nicati, K. Toyama, H.J. Shaw, J. Lightwave Technol. 13, 7 (1995)

5 Y. Shen, X. Zhang, K. Chen, J. Lightwave Technol. 23, 5 (2005)

6 K. Ogusu, IEEE Photon. Technol. Lett. 14, 7 (2002)

7 K. Brown, A.W. Brown, B.G. Colpitts, Opt. Fiber Technol. 11, 131 (2005) 